\title{
Inhibitor of protein synthesis phase shifts a circadian pacemaker in mammalian SCN
}

\author{
SHIN-ICHI T. INOUYE, JOSEPH S. TAKAHASHI, \\ FRANZISKA WOLLNIK, AND FRED W. TUREK \\ Department of Neurobiology and Physiology, Northwestern University, Evanston, Illinois 60208
}

\begin{abstract}
InOUye, Shin-IChi T., Joseph S. TAKahashi, Franziska WOLLNIK, AND FRED W. TUREK. Inhibitor of protein synthesis phase shifts a circadian pacemaker in mammalian SCN. Am. J. Physiol. 255 (Regulatory Integrative Comp. Physiol. 24): R1055-R1058, 1988.-The suprachiasmatic nucleus (SCN) of the hypothalamus contains a circadian pacemaker that regulates many circadian rhythms in mammals. Experimental work in microorganisms and invertebrates suggests that protein synthesis is required for the function of the circadian oscillator, and recent experiments in golden hamsters suggest an acute inhibition of protein synthesis can induce phase shifts in a mammalian circadian pacemaker. To determine whether protein synthesis in the SCN region is involved in the generation of circadian rhythms in mammals, a protein synthesis inhibitor, anisomycin, was microinjected into the $\mathrm{SCN}$ region, and the effect on the circadian rhythm of locomotor activity of hamsters was measured. A single injection of anisomycin into the SCN region induced phase shifts in the circadian activity rhythm that varied systematically as a function of the phase of injection within the circadian cycle. These results suggest that protein synthesis may be involved in the generation of circadian rhythms in mammals and that the anatomic site of action of anisomycin is within the hypothalamic suprachiasmatic region.
\end{abstract}

biological rhythms; biological clocks; anisomycin; golden hamsters

THE PERIODIC CHANGE in the physical environment throughout the solar day has led to the evolution of circadian oscillators that function as biological clocks in most, if not all, eukaryotic organisms. The search for the anatomic location of the circadian clock in mammals has focused on the suprachiasmatic nucleus (SCN) of the hypothalamus $(13,18,24,26)$. Destruction of these bilaterally paired nuclei leads to the loss of various circadian rhythms in rodents, including the rhythm of wheelrunning activity in hamsters (17). The abolition of circadian rhythms after SCN lesions is also seen in primates (monkeys) (1) and birds (22). In rats, the persistence of neural circadian rhythmicity in SCN tissue surgically isolated from the rest of the brain $(8,9)$, as well as in in vitro preparations $(3,5,7,19)$, also suggests that the SCN contains an autonomous oscillator capable of generating circadian signals.

In microorganisms and invertebrates, protein synthesis on $80 \mathrm{~S}$ ribosomes appears to be involved in the generation of circadian rhythms because administration of inhibitors of protein synthesis causes phase shifts in circadian rhythms $(10-12,15)$. We have previously demonstrated that the systemic administration of two protein synthesis inhibitors, anisomycin (12 mg/injection) or cycloheximide (10 mg/injection), are both effective in inducing phase shifts in the circadian activity rhythm of hamsters housed in constant darkness (23). The magnitude and direction of the phase shifts are dependent on the circadian time of drug treatment. As a first step in determining the anatomic location at which inhibition of protein synthesis induces phase shifts in mammalian circadian rhythms, we examined the effects on the circadian rhythm of activity of microinjecting microgram quantities of anisomycin, a reversible $80 \mathrm{~S}$ ribosomal protein synthesis inhibitor (6), directly into the hypothalamic SCN region of hamsters.

\section{METHODS}

Adult male golden (Syrian) hamsters (Lakeview Hamster Colony, Newfield, NJ), maintained on a 14:10-h light-dark cycle, were stereotaxically implanted under pentobarbital sodium anesthesia $(60 \mathrm{mg} / \mathrm{kg})$ with a microinjection guide assembly aimed at the SCN region. The microinjection guide assembly consists of an outer guide tube (23 gauge) and an inner stainless wire pin ( 0.3 $\mathrm{mm}$ diam), the tip of which is set to extend $1.0 \mathrm{~mm}$ farther beyond the outer guide cannula. The tip of the injection needle (30 gauge), which is connected to a microsyringe drive through polyethylene tubing, is designed to extend $0.7 \mathrm{~mm}$ beyond the outer guide cannula when an injection is made. These configurations leave at the tip of the injection needle a small cylindrical space $\sim 0.3 \mathrm{~mm}$ in length and $0.3 \mathrm{~mm}$ in diameter into which the drug is injected. The stereotaxic coordinates of the microinjection guide assembly were $0.2 \mathrm{~mm}$ posterior to bregma, $1.3 \mathrm{~mm}$ lateral to midline with the cannula at a $9^{\circ}$ angle, and $-7.6 \mathrm{~mm}$ ventral from the dura.

After stereotaxic surgery, the animals were blinded to prevent the possibility of light-induced phase shifts in the circadian activity rhythm. Once a stable free-running rhythm of locomotor activity was established, the animals received a microinjection of $5 \mu \mathrm{g}$ of anisomycin dissolved in saline $(1 \mu \mathrm{l})$, or saline alone, into the SCN region through the chronically implanted cannula. The phase-shifting effects of anisomycin on the free-running rhythm of locomotor activity were determined by estimating the steady-state phase of the daily onsets of activity before and after the injection as described in 


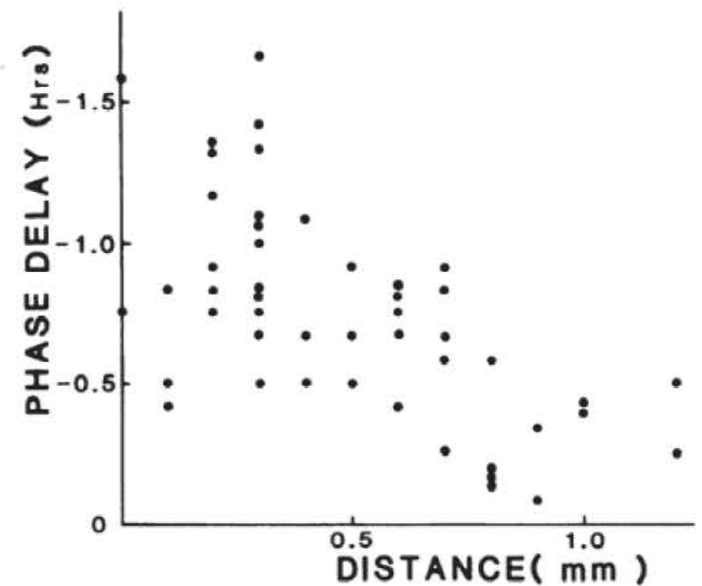

FIG. 1. Plot of phase delay in activity rhythm induced by a single injection of $5 \mu \mathrm{g}$ of anisomycin presented $12 \mathrm{~h}$ after onset of activity in hamsters as a function of distance of cannula tip from suprachiasmatic nucleus.

Daan and Pittendrigh (2). The precise nature of the onset of wheel-running activity in the hamster makes it easy to determine phase shifts as small as $10 \mathrm{~min}$ in this rhythm.

At the end of the experiment, animals were perfused transcardially, and the brains were examined histologically to determine the location of the injection site. In a pilot study, we observed that phase shifts in the activity rhythm to a 5- $\mu \mathrm{g}$ dose of anisomycin presented at circadian time 0 were only reliably observed when the cannula tip was between 0 and $0.6-0.7 \mathrm{~mm}$ from the SCN (Fig. 1 ). Therefore, in determining the phase-response curve to microinjections of anisomycin, only those animals in which the injection site was within $0.5 \mathrm{~mm}$ of the SCN were included in the analysis of the data in order to be sure that the injection of anisomycin was close enough to the SCN to be effective.

\section{RESULTS}

Administration of anisomycin into the SCN produced phase shifts in the activity rhythm that were dependent on the circadian time (CT) of the injection. The direction and magnitude of phase shifts produced by anisomycin microinjected into the $\mathrm{SCN}$ region at various times throughout the circadian cycle are illustrated in Fig. 2. Injections of anisomycin at phases between CT 14 and CT 4 (CT 12 is by convention represented by the onset of locomotor activity in the nocturnal hamster) consistently caused phase delays of $\sim 1 \mathrm{~h}$ in the activity rhythm, whereas injections at phases between CT 6 and CT 10 caused phase advances of a similar magnitude. The activity records of representative animals are shown in Fig. 3 to demonstrate both a phase delay (Fig. $3 A$ ) and a phase advance (Fig. $3 B$ ) in response to the anisomycin. Microinjection of saline into the SCN region had no consistent effects on the activity rhythm (Fig. $3 C$ ) regardless of the time of administration (Fig. 2 C).

\section{DISCUSSION}

The results from these experiments suggest that protein synthesis may be involved in the circadian time-
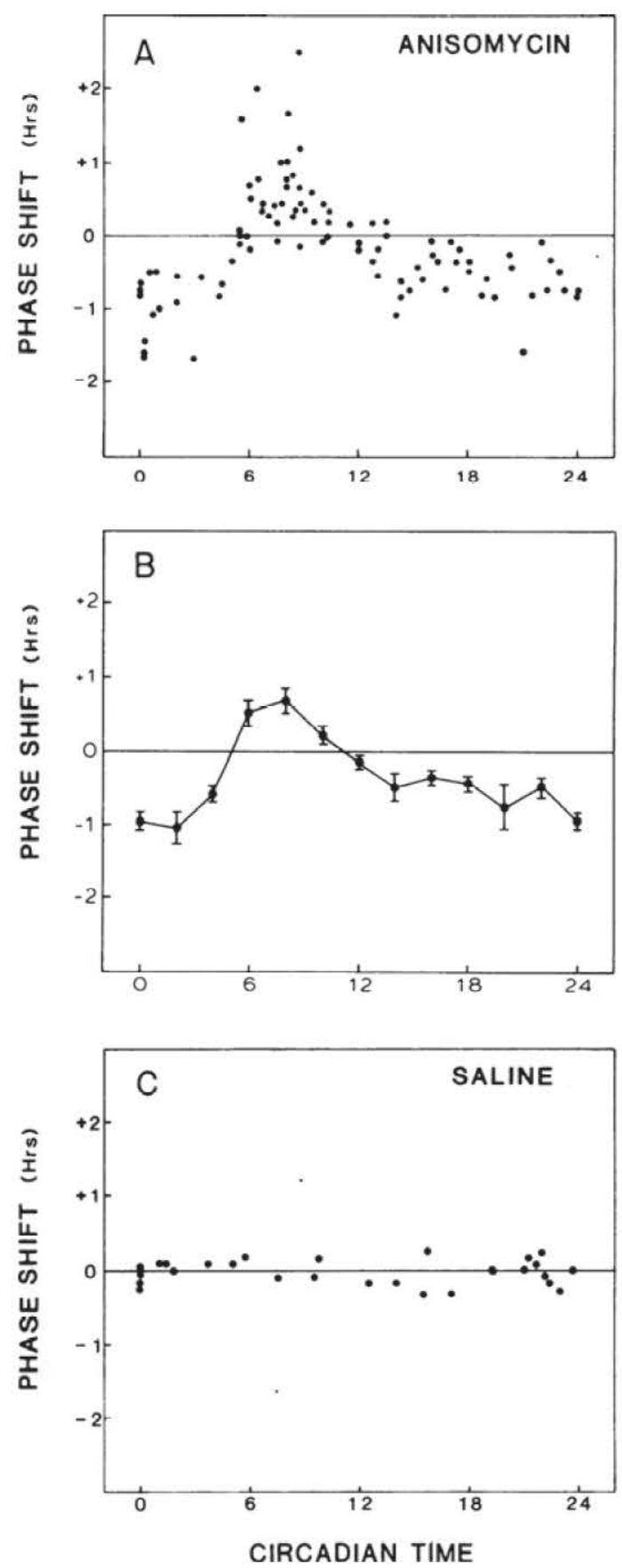

FIG. 2. A: phase dependence of effects on locomotor rhythm of anisomycin $(5 \mu \mathrm{g})$ microinjections at various circadian times into suprachiasmatic nucleus region of hamsters. Each point represents phase shift induced in free-running activity rhythm by a single microinjection of anisomycin at time indicated on horizontal axis. Circadian time (CT) 12 represents time of onset of locomotor activity. Advances are plotted as positive values and delays as negative values. $B$ : phaseresponse curve for anisomycin microinjections. Each point represents mean \pm SE phase shift plotted in 2-h bins. Mean phase shifts were significantly different from zero at CT 0 and $8, P<0.001$; CT 16 and $18, P<0.01$; CT 2, 4, 6, 10, and 22, $P<0.05$ (Student's $t$ test). Values presented for CT 0 and CT 24 represent same data. $C$ : saline microinjections did not cause phase shifts at any phase. Each point represents phase shift induced by a single saline microinjection.

keeping mechanism in the SCN. The shape of the phaseresponse curve (PRC) to microinjections of $5.0 \mu \mathrm{g}$ anisomycin directly into the $\mathrm{SCN}$ region (Fig. $2 B$ ) is very similar in shape to the PRC generated by subcutaneous 

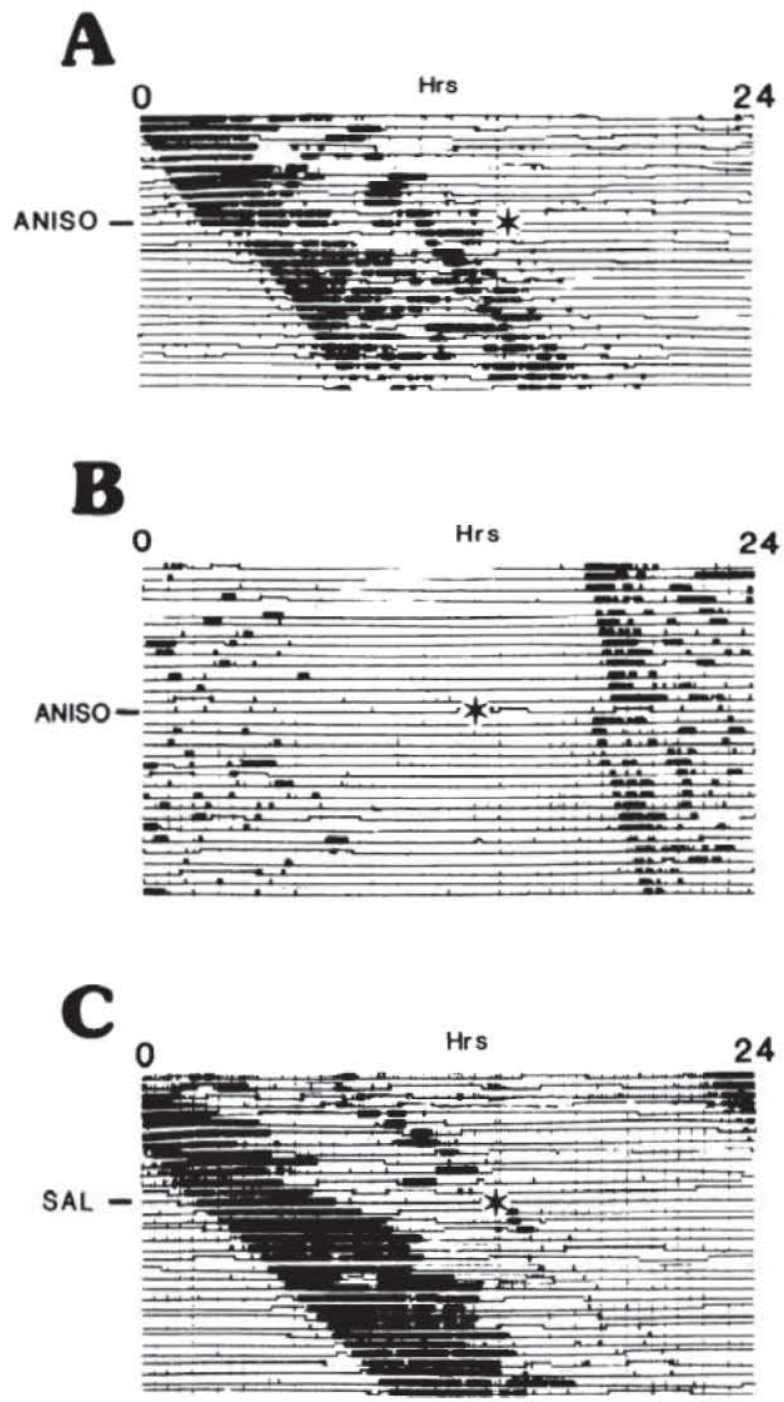

FIG. 3. Representative activity records of free-running hamsters microinjected with either anisomycin (ANISO) or saline (SAL) into suprachiasmatic nucleus region. Each horizontal line represents activity record over a single 24 -h period and successive days are plotted beneath each other. Stars represent times of an injection. $A$ : microinjection of $5 \mu \mathrm{g}$ anisomycin at circadian time (CT) 0 induced a phase delay in activity rhythm. B: microinjection of $5 \mu \mathrm{g}$ anisomycin at CT 6.5 induced a phase advance of the rhythm. $C$ : microinjection of $1 \mu \mathrm{l}$ saline at CT 0 did not induce a phase shift in rhythm.

injections of $12 \mathrm{mg}$ anisomycin (23). Thus the effectiveness of microgram quantities of anisomycin administered into the SCN region in phase shifting the circadian rhythm of activity is equivalent to the effects of systemic administrations with doses more than three orders of magnitude greater. Preliminary data from a dose-response curve study indicate that the maximum phase shift that can be induced by inhibition of protein synthesis is $\sim 1-2 \mathrm{~h}$ (Inouye, unpublished results).

Although we have not excluded the possibility that the phase-dependent phase shifts of the circadian system are due to unknown side effects of anisomycin, this seems unlikely in view of the results of recent experiments that examined the effects of related drugs on the circadian activity rhythm of hamsters (23). Both anisomycin and cycloheximide decrease catecholamine biosynthesis by inhibiting tyrosine hydroxylase activity (20), and both also inhibit acetylcholinesterase activity $(28,29)$. Nevertheless, peripheral injections of $\alpha$-methyl-p-tyrosine, an inhibitor of tyrosine hydroxylase (4), and physostigmine, an inhibitor of acetylcholinesterase (4), were not effective in phase shifting the activity rhythm of hamsters (23). Furthermore, subcutaneous injections of anisomycin or cycloheximide are both effective in inducing phase shifts in the circadian activity rhythm of hamsters despite the different mechanisms through which protein synthesis is inhibited by these agents (23).

It is not clear whether the phase shifts induced by anisomycin are due to a general reduction of protein synthesis or alternatively to an inhibition of a specific protein(s) that may be involved in oscillatory function. However, in either case, because the phase shifts were permanent, anisomycin is acting on the circadian oscillator either directly or indirectly through an input pathway to the oscillator (24). The presence of both phase delays and phase advances in the PRC also suggests that the effects of anisomycin are due to an action on a specific component of the circadian clock mechanism rather than the result of some nonspecific effect on metabolism.

The PRC for the circadian rhythm of locomotor activity in hamsters to anisomycin injections is quite different from the PRC to light pulses $(2,21)$, indicating that anisomycin is perturbing the circadian clock through a mechanism different from light. Interestingly, in hamsters the PRC to injections of anisomycin is qualitatively similar to those generated by injections of a short-acting benzodiazepine, triazolam, under conditions of either constant light or constant dark (27). However, at the present time it is not known whether the phase-shifting effects of protein synthesis inhibitors and benzodiazepines involve similar mechanisms.

Mrosovsky (14) has obtained PRCs to cage changing and social interactions in hamsters that are similar in shape to those observed for anisomycin and triazolam. He has suggested that phase shifts in the circadian oscillator in response to drug treatment may be due in part to an alteration in the general arousal state of the organism. Indeed, exposure to a running wheel for a short period of time by itself can induce phase shifts in the activity rhythm of hamsters that are similar to those observed after treatment with anisomycin or triazolam (Wickland and Turek, unpublished results). However, it does not appear that phase shifts induced by injections of anisomycin are mediated through an increase in locomotor activity. In the present study, an increase in activity for $>30 \mathrm{~min}$ in response to injections of anisomycin was only observed in $\sim 20 \%$ of the animals and no significant correlation between amount of activity, and phase shift was observed for any of the circadian times tested. Although only wheel-running behavior was recorded in the present study, visual observations of the animals indicate that there is no general increase in activity associated with injections of anisomycin.

PRCs to pulse administration of protein synthesis inhibitors have been determined for a number of organisms, including Aplysia (10-12, 16), Gonyaulax (25), and Neurospora (15). It is intriguing to note that the PRC to 
anisomycin injected into the hamster SCN region has qualitative similarities with the PRCs to protein synthesis inhibitors in Aplysia and Neurospora. The delay regions of the PRCs for anisomycin in Aplysia and for cycloheximide in Neurospora both peak near CT 0 . The advance regions are more variable. In Aplysia, anisomycin produces either very small or undetectable phase advances near CT 6; whereas in Neurospora cycloheximide produces clear advances that peak at CT 6. Perhaps more importantly, the "breakpoints" (i.e., the transition from phase delays to phase advances) of the PRCs are clustered around CT 4 in Aplysia and Neurospora as well as in the hamster. These similarities may suggest that the molecular mechanisms generating circadian rhythms in the mammalian SCN share some common features with those found in diverse phylogenetic groups.

This work was supported by National Science Foundation Presidential Young Investigator award DCB-8451642 and Searle Scholars award 85-H-107 (to J. S. Takahashi), National Institute of Mental Health Grants MH-41211 and MH-39592, and National Institute of Child Health and Human Development Grant HD-09885.

Present address of S.-I. T. Inouye: Mitsubishi-Kasei Institute of Life Sciences, 11, Minami-Ooya, Machida-shi, Tokyo 194, Japan.

Address for reprint requests: F. W. Turek, Dept. of Neurobiology and Physiology, Northwestern Univ., Evanston, IL 60208.

Received 20 January 1988; accepted in final form 15 July 1988.

\section{REFERENCES}

1. Albers, H. E., R. Lydic, P. H. Gander, ANd M. C. Moore-Ede. Role of the suprachiasmatic nuclei in the circadian timing system of the squirrel monkey. I. The generation of rhythmicity. Brain Res. 300: 275-284, 1984.

2. DAAN, S., AND C. S. PitTendrigh. A functional analysis of circadian pacemakers in nocturnal rodents. II. The variability of phase response curves. J. Comp. Physiol. 106: 253-266, 1976.

3. EARNEST, D. J., AND C. D. SLADEK. Circadian rhythms of vasopressin release from individual rat suprachiasmatic explants in vitro. Brain Res. 382: 129-133, 1986.

4. Gilman, A. G., L. S. Goodman, T. W. Rall, and F. Murad (Editors). The Pharmacological Basis of Therapeutics. New York: Macmillan, 1985.

5. Green, D. J., AND R. GilletTe. Circadian rhythm of firing rate recorded from single cells in the rat suprachiasmatic brain slice. Brain Res. 245: 198-200, 1982.

6. Grollman, A. P., And M.-T. Huang. Protein Synthesis. New York: Dekker, 1976, p. 125-167.

7. Groos, G., AND J. HeNDRIKs. Circadian rhythms in electrical discharge of rat suprachiasmatic neurones recorded in vitro. Neurosci. Lett. 34: 283-288, 1982.

8. InOUYe, S. T., AND H. Kawamura. Persistence of circadian rhythmicity in a mammalian hypothalamic "island" containing the suprachiasmatic nucleus. Proc. Natl. Acad. Sci. USA 76: 5962-5966, 1979.
9. INOUYE, S. T., AND H. KaWAmura. Characteristics of a circadian pacemaker in the suprachiasmatic nucleus. J. Comp. Physiol. 146: $153-160,1982$.

10. JACKLET, J. W. Neuronal circadian rhythms: phase shifting by a protein synthesis inhibitor. Science Wash. DC 198: 69-71, 1977.

11. JACKLET, J. W. Neural organization and cellular mechanisms of circadian pacemakers. Int. Rev. Cytology 49: 251-294, 1984.

12. LOTSHAW, D. P., AND J. W. JACKLET. Involvement of protein synthesis in circadian clock of Aplysia eye. Am. J. Physiol. 250 (Regulatory Integrative Comp. Physiol. 19): R5-R17, 1986.

13. MOORE, R. Y. Organization and function of a central nervous system circadian oscillator: the suprachiasmatic hypothalamic nucleus. Federation Proc. 42: 2783-2789, 1983.

14. Mrosovsky, N. Phase response curves for social entrainment. $J$. Comp. Physiol. 163: 35-46, 1988.

15. Nakashima, H., J. Perlman, and J. F. Feldman. Cycloheximideinduced phase shifting of circadian clock of Neurospora. Am. J. Physiol. 241 (Regulatory Integrative Comp. Physiol. 10): R31-R35, 1981.

16. Rothman, B. S., AND F. Strumwasser. Phase-shifting the circadian rhythm of neural activity in the isolated Aplysia eye with puromycin and cycloheximide: electrophysical and biochemical studies. J. Gen. Physiol. 68: 359-384, 1976.

17. RUSAK, B. The role of the suprachiasmatic nuclei in the generation of circadian rhythms in the golden hamster. J. Comp. Physiol. 118: 145-164, 1977.

18. RUSAK, B., AND I. ZUCKER. Neural regulation of circadian rhythms. Physiol. Rev. 59: 449-526, 1979.

19. Shibata, S., Y. Oomura, H. Kita, and K. Hattori. Circadian rhythmic changes of neuronal activity in the suprachiasmatic nucleus of the rat hypothalamic slice. Brain Res. 247: 154-158, 1982.

20. Squire, L. R., R. Kuczenski, and S. H. Barondes. Tyrosine hydroxylase inhibition by cycloheximide and anisomycin is not responsible for their amnesic effect. Brain Res. 82: 241-248, 1974.

21. Takahashi, J. S., P. J. DeCoursey, L. Bauman, and M. MenAKER. Spectral sensitivity of a novel photoreceptive system mediating entrainment of mammalian circadian rhythms. Nature Lond. 308: 186-188, 1984.

22. TAKAHASHI, J. S., AND M. MENAKER. Role of the suprachiasmatic nuclei in the circadian system of the house sparrow, Passer domesticus. J. Neurosci. 2: 815-828, 1982.

23. TAKAhashi, J. S., AND F. W. TUREK. Anisomycin, an inhibitor of protein synthesis, perturbs the phase of a mammalian circadian pacemaker. Brain Res. 405: 199-203, 1987.

24. TAKAHASHI, J. S., AND M. ZATZ. Regulation of circadian rhythmicity. Science Wash. DC 217: 1104-1111, 1982.

25. TAYLOR, W. R., J. C. DunlaP, AND J. W. Hastings. Inhibitors of protein synthesis on $80 \mathrm{~S}$ ribosomes phase shift the Gonyaulax clock. J. Exp. Biol. 97: 121-136, 1982.

26. TUREK, F. W. Circadian neural rhythms in mammals. Annu. Rev. Physiol. 47: 49-64, 1985.

27. TUREK, F. W., AND S. LOSEE-OLSON. A benzodiazepine used in the treatment of insomnia phase-shifts the mammalian circadian clock. Nature Lond. 321: 167-168, 1986.

28. ZECH, R., AND G. F. DormagK. Puromycin and cycloheximide as inhibitors of human brain acetylcholinesterase. Brain Res. 86: 339342,1975 .

29. ZECH, R., AND G. F. DORMAGK. Inhibition of acetylcholinesterase by anisomycin. Life Sci. 19: 157-162, 1976. 